\title{
Tests and calibrations of nuclear track detectors (CR39) for operation in high neutron flux
}

\author{
E. E. Kading, ${ }^{1}$ O. Aviv, ${ }^{2}$ I. Eliyahu, ${ }^{2}$ M. Gai $\odot,{ }^{1,}{ }^{*}$ S. Halfon, ${ }^{2}$ M. Hass,,${ }^{3, \dagger}$ C. R. Howell,${ }^{4}$ D. Kijel, ${ }^{2}$ Y. Mishnayot,${ }^{2}$ I. Mukul, ${ }^{3}$ \\ A. Perry, ${ }^{2}$ Y. Shachar, ${ }^{3}$ Ch. Seiffert, ${ }^{5}$ A. Shor, ${ }^{2}$ I. Silverman, ${ }^{2}$ S. R. Stern, ${ }^{1}$ Th. Stora, ${ }^{5}$ D. R. Ticehurst,${ }^{4}$ \\ A. Weiss, ${ }^{6}$ and L. Weissman ${ }^{2}$ \\ ${ }^{1}$ LNS at Avery Point, University of Connecticut, Groton, Connecticut 06340, USA \\ ${ }^{2}$ Soreq Nuclear Research Center, Nuclear Physics Engineering Division, Yavne 81800, Israel \\ ${ }^{3}$ Department of Particle Physics, Weizmann Institute of Science, Rehovot 76100, Israel \\ ${ }^{4}$ TUNL, Department of Physics, Duke University, Durham, North Carolina 27708-0308, USA \\ ${ }^{5}$ European Organization for Nuclear Research (CERN), CH-1211 Geneva, Switzerland \\ ${ }^{6}$ Faculty of Engineering, Bar Ilan University, Ramat Gan 52900, Israel
}

(Received 6 October 2019; accepted 21 April 2020; published 3 June 2020)

\begin{abstract}
The neutron beams produced by the Soreq Applied Research Accelerator Facility (SARAF) operating with the liquid lithium target (LiLiT) present scientific opportunities in nuclear astrophysics. Of interest are measurements (in our setup) of 1.5 to $3.0 \mathrm{MeV}$ alpha-particles and $1.5 \mathrm{MeV}$ protons, emanating from the interaction of neutrons with ${ }^{7} \mathrm{Be}$. The deduced cross sections, are important for understanding the cosmological "Primordial ${ }^{7}$ Li Problem." However, major experimental challenges arise when measuring such low-energy charged particles due to the high flux neutron beams produced by the LiLiT (up to $\sim 5 \times 10^{10} \mathrm{n} / \mathrm{sec} / \mathrm{cm}^{2}$ ). We present a study of the operation of CR39 nuclear track detectors (NTD), in such a high neutron flux. The CR39 plates were energy calibrated with $3.18 \mathrm{MeV}$ alpha-particles from a ${ }^{148} \mathrm{Gd}$ standard radioactive source, and by using Rutherford backscattering of $1.5 \mathrm{MeV}$ alpha-particles as well as $1.5 \mathrm{MeV}$ protons from a thin $\left(100 \mu \mathrm{g} / \mathrm{cm}^{2}\right)$ gold foil. The plates were etched in a $6.25 \mathrm{~N} \mathrm{NaOH}$ solution for 30 minutes at $90^{\circ} \mathrm{C}$ to produce micron-sized circular pits. The alpha $(3.18 \mathrm{MeV})$ and proton $(1.5 \mathrm{MeV})$ etch efficiencies were measured to be $93.3 \pm 6.1 \%$ and $8.7 \pm 1.3 \%$, respectively. After etching, the plates were scanned with a fully automated microscope. A segmentation algorithm that addressed the challenges posed by the intense neutron beam was developed. A measurement of the interaction of cold neutrons with CR39 showed that the ${ }^{17} \mathrm{O}(n, \alpha){ }^{14} \mathrm{C}$ reaction inside the CR39 was a major source of background. Since the tracks were formed inside the CR39, this background extended up to the full energy deposit of $\sim 2.1 \mathrm{MeV}$. This neutron-beam-induced background inside the CR39 plates, observed here for the first time, will most likely be the limiting factor for observing $\sim 2 \mathrm{MeV}$ alpha-particles with CR39, in measurements of small cross sections (below a few $\mathrm{mb}$ ) of $(n, \alpha)$ reactions. However, in measurements of larger cross sections, this ${ }^{17} \mathrm{O}(n, \alpha)$ "background line" can be used as an internal self-calibration of alpha-particles with energies up to $\sim 2.1 \mathrm{MeV}$, and thus allow to correct for piece-to-piece variations in the energy-to-track size calibration of CR39 plates.
\end{abstract}

DOI: 10.1103/PhysRevResearch.2.023279

\section{INTRODUCTION}

\section{A. SARAF}

The Soreq Applied Research Accelerator Facility (SARAF) [1], at the Soreq Nuclear Research Center (SNRC) in Israel, offers major research opportunities for studies of neutron interactions that are important for stellar evolution theory and cosmology. In particular, as discussed in [2], the "physics goal" of this project is measuring the cross section for the interaction of neutrons with ${ }^{7} \mathrm{Be}$, in the ${ }^{7} \mathrm{Be}(n, \alpha)$,

\footnotetext{
*Corresponding author: moshe.gai@uconn.edu

${ }^{\dagger}$ Deceased.
}

Published by the American Physical Society under the terms of the Creative Commons Attribution 4.0 International license. Further distribution of this work must maintain attribution to the author(s) and the published article's title, journal citation, and DOI.
${ }^{7} \operatorname{Be}(n, \gamma \alpha)$, and ${ }^{7} \operatorname{Be}(n, p)$ reactions, that are essential for understanding the cosmological lithium problem $[3,4]$.

In a measurement of the interaction of neutron with ${ }^{7} \mathrm{Be}$, one needs to measure $1.5 \mathrm{MeV}$ protons from the ${ }^{7} \mathrm{Be}(n, p)$ reaction, and high-energy alpha-particles at $9.5 \mathrm{MeV}$ from the ${ }^{7} \mathrm{Be}(n, a)$ reaction and $\sim 8.4 \mathrm{MeV}$ from the ${ }^{7} \mathrm{Be}\left(n, \gamma_{3,4} \alpha\right)$ reaction, as well as low-energy " $1.5 \mathrm{MeV}$ " alpha-particles from the ${ }^{7} \mathrm{Be}\left(n, \gamma_{1} \alpha\right)^{8} \mathrm{Be}^{*}(3.03)$ reaction [4]. We note that the decay of the broad $(\Gamma=1.5 \mathrm{MeV}) 2^{+}$state of ${ }^{8} \mathrm{Be}$ at $3.03 \mathrm{MeV}$, leads to a broad distribution of alphaparticles, beyond $1.5 \mathrm{MeV}$. Since the thermal neutron cross section of the ${ }^{7} \mathrm{Be}(n, p)$ reaction is known to be very large ( $\sim 50000$ barns), one needs to reduce the proton rate in such measurements.

In our setup, the measurement of the high-energy alphaparticles $(9.5$ and $\sim 8.4 \mathrm{MeV}$ ) was carried out with a foil to stop the $1.5 \mathrm{MeV}$ protons. This foil degraded the highenergy alpha-particles to 1 to $3 \mathrm{MeV}$, but it also stopped the "1.5 MeV" alpha particles. For the measurement of the 
"1.5 MeV" alpha-particles, we removed the stopper foil, but in this case we need to reduce the efficiency for measuring $1.5 \mathrm{MeV}$ protons, as we report here. We note that still with the reduced proton detection efficiency, we observed a $1.5 \mathrm{MeV}$ proton signal above background, from the ${ }^{7} \mathrm{Be}(n, p)$ reaction, with a sufficient accuracy of $\pm 10 \%$, as we report in [2]. Accordingly, at $49.5 \mathrm{keV}$ we measure the cross section for emanating $1.5 \mathrm{MeV}$ protons which is approximately a factor 600 larger than the cross section of emanating "1.5 MeV" alpha-particle, but the observed yield of the $1.5 \mathrm{MeV}$ protons is only a factor 30 larger (with two alpha-particles per decay), as shown in Fig. 3 of [2].

Thus, in the experimental setup, with the ${ }^{7} \mathrm{Be}$ target, discussed in [2], that is also discussed in this paper, only 1 to $3 \mathrm{MeV}$ alpha-particles and $1.5 \mathrm{MeV}$ protons are needed to be measured. We chose to use nuclear track detectors (NTD) made of CR39 to detect these charged particles, and we report here on energy calibration of CR39 for detecting 1.5 $\mathrm{MeV}$ protons and 1.5 to $3.0 \mathrm{MeV}$ alpha-particles. We demonstrate that the measured alpha-particles and protons are fully characterized by the radii of the corresponding pits in the etched CR39 plates. We reveal, for the first time, a background due to the interaction of neutrons with the CR39 itself. But we show that this background can be used as a $\sim 2 \mathrm{MeV}$ "self-calibration line" to measure the cross sections of the ${ }^{7} \mathrm{Be}(n, \alpha)$ reaction.

The neutron beams produced at the SARAF (in phase I) by the liquid lithium target (LiLiT) [5-7] via the thick-target ${ }^{7} \mathrm{Li}(p, n){ }^{7} \mathrm{Be}$ reaction near threshold $(1.88 \mathrm{MeV})$, have a quasi-Maxwellian energy distribution peaked at tens of $\mathrm{keV}$ $[8,9]$, and thus it mimics cosmological and stellar conditions, as shown in Fig. 1 of [2]. However, the resulting high neutron flux, that can be as large as $\sim 5 \times 10^{10} \mathrm{n} / \mathrm{sec} / \mathrm{cm}^{2}$, presents a major challenge for detector systems, even for detectors that can survive in such a "hostile" neutron environment. The neutrons are produced with $E_{p}=1.91-1.95 \mathrm{MeV}$ via the ${ }^{7} \mathrm{Li}(p, n)$ reaction, with an "effective temperature" $\mathrm{kT}=$ $20-50 \mathrm{keV}$ (energies spread from 1 to $180 \mathrm{keV}$ ). They are well suited for measurement of neutron-induced $(n, \alpha)$ reactions which are important in astrophysical scenarios. It was shown by the MIT group [10,11] (in extensive measurements at the NIF facility in the Lawrence Livermore National Lab and the Omega facility in Rochester, NY), that CR39 NTD are well suited for measurements in a high flux neutron environment of $\mathrm{MeV}$ neutrons, hence, motivating the use of CR39 NTD with lower-energy neutrons, as we report here.

\section{B. CR39 nuclear track detector}

The intense neutron background precludes the use of standard spectroscopic tools, such as silicon detectors, to measure the energies of protons and alpha-particles from the interaction of neutrons with the ${ }^{7} \mathrm{Be}$ target. We are currently pursuing the use of diamond detectors [12], which are known to be good spectroscopic tools that are robust against the large neutron flux. However, diamond detectors were not used in the current study. In this (feasibility) study we tested CR39 plates for measuring the energy of 1.5 protons and 1.5 to $3.0 \mathrm{MeV}$ alpha-particles, of interest. The CR39 plates (Columbia Resin $\# 39$, poly allyl diglycol carbonate - $\left.\mathrm{PADC}, \mathrm{C}_{12} \mathrm{H}_{18} \mathrm{O}_{7}\right)$ were purchased from Homalite [13] and tested in the high neutron flux produced by the LiLiT at SARAF.

Charged particles, when traversing a CR39 plate, leave behind a track of radiation damage, consisting of broken PADC molecules. When properly etched, as shown below, the etching agent removes a portion of the CR39 plate (commonly referred to as etch rate $V_{b}$ ); however, the etch rate along the track $\left(V_{t}\right)$ is considerably faster, leading to the formation of a pit. The pits in the etched NTD produce two different types of images when viewed with a standard transmitted-light brightfield microscope (depending on the focus plane). With our etching conditions, the measured pits are either a dark spot where the light is scattered away by the dip, or have a bright focused spot of light in the center. This led us to develop the sophisticated system and algorithm we discuss below, where a z-stack of 11 images, separated by 1.5 microns was acquired, using the Bar Ilan University (BIU) automated microscope system.

Neutron yield measurements [10,11] of MeV fast neutrons, demonstrate the viability of CR39 NTD for cross section measurements in a high flux neutron environments. In the same time, measurements with CR39 NTD to detect charged particles from the ${ }^{10} \mathrm{~B}(n, \alpha){ }^{7} \mathrm{Li}$ reaction [14] (with keV neutrons and a total integrated neutron-number of $\left.4.6 \times 10^{9}\right)$, also demonstrated the viability of CR39 for measurements of cross sections with $\mathrm{keV}$ epithermal neutrons.

We chose to analyze and measure the radii of the dark pit images to ascertain the energy deposited by the protons and alpha-particles in the CR39 detectors. The etch rate of the track is directly proportional to the energy loss along the track $(\mathrm{dE} / \mathrm{dx})$ of the charged particle, and thus no simple monotonic relationship exists between the energy of the incoming particle and the pit radius [15]. Indeed, large variations in the dependence of the pit radii on the energy of alpha-particles (and protons) were observed for different etch conditions [15]. As shown in Fig. 2 of [15], for some etch conditions, the observed radii exhibit a "Bragg-like" dependence, not a simple monotonic dependence.

Hence, in this study we calibrate our CR39 plates only over the narrow energy region of interest. But, since the formation of the pit depends on the radiation damage $(\mathrm{dE} / \mathrm{dx})$ of the charged particle and on the etch conditions, a careful and self-consistent strict etch protocol is required, as we discuss below.

We note that, for a charged particle that enters the CR39 with a certain energy, the etched volume samples $\mathrm{dE} / \mathrm{dx}$ along the track of the charged particle. However, for charged particles originating from interactions inside the CR39, the etched volume may contain a fraction of the track, and in this case, we sample a fraction of the full energy deposited in the CR39. Thus, for charged particles generated inside the CR39 plate, we measure pits corresponding to energies up to the full energy of the particles.

\section{Goal}

The goal of this study is to measure (in our experimental setup) $1.5 \mathrm{MeV}$ protons and 1.5 to $3.0 \mathrm{MeV}$ alpha-particles from the interaction of epithermal neutrons with ${ }^{7} \mathrm{Be}$. To achieve this goal we characterize the in-beam induced background of epithermal neutrons interacting inside the NTD- 
CR39 plates. We demonstrate for the first time, that this background is dominated by the ${ }^{17} \mathrm{O}(n, \alpha)$ reaction inside the CR39 plates. We describe the automated acquisition process that enabled us to produce images in which all of the objects are dark spots. Briefly, this is done by acquiring a stack of eleven images in different focus planes, and then either producing a minimum intensity projection of the stack, or stitching the entire three-dimensional (3D) stack together, then selecting the focus plane with the sharpest dark objects.

\section{EXPERIMENTAL PROCEDURES AND RESULTS}

We report on several measurements with different setups in different labs: research and devlopment studies at the TUNL at Duke University, energy calibrations measured at the LNS of the University of Connecticut, and at the $3 \mathrm{MV}$ van de Graaff at the Weizmann Institute of Science. We also include measurements with cold neutrons at the ILL in Grenoble, and in beam measurements with epithermal neutrons at the SARAF. The etched CR39 were analyzed at the microscopy laboratory of the Bar Ilan University. For the "smooth" flow of the reading, we organize the paper with a discussion of each facility used in this project.

For all these measurements we used 50 CR39 plates that were cut from the same large "page" (H-911) distributed by Plastic Products and produced by Homalite [13]. Hence, all CR39 plates used in this extensive study are from the same "batch." A measurement of the background (intrinsic noise) of the CR39 plate was performed on line by studying the pits behind an absorber foil that stopped all charged particles. In the initial research and development phase of this study, we also tested TASL plates [16], but they were not used in this measurement.

\section{A. Energy calibration}

The CR39 plates were energy calibrated with alphaparticles from a standard spectroscopic ${ }^{148} \mathrm{Gd}$ radioactive source $(3.18 \mathrm{MeV})$. In addition, we used the $3.0 \mathrm{MeV}$ van de Graaff accelerator of the Weizmann Institute of Science (WIS) to calibrate the energy deposited in the CR39 plates, using the setup shown in Fig. 1, with Rutherford backscattering (RBS) off a thin $\left(100 \mu \mathrm{g} / \mathrm{cm}^{2}\right)$ gold foil, of $1.5 \mathrm{MeV}$ alpha-particle and $1.5 \mathrm{MeV}$ protons.

Prior to these calibration measurements extensive studies for optimizing the etch conditions were performed at the Laboratory for Nuclear Science (LNS) at the University of Connecticut, using standard radioactive sources $\left({ }^{148} \mathrm{Gd}\right.$, ${ }^{241} \mathrm{Am}$, and $\left.{ }^{228} \mathrm{Th}\right)$. In addition, beams of alpha particles $(1.2$ to $9.0 \mathrm{MeV}$ ) and protons (1.5 MeV), extracted from the TUNL tandem accelerator at Duke University were also used in the initial research and development stages for optimizing the etching conditions $\left(70\right.$ to $90^{\circ} \mathrm{C}$ ) to yield the desired smaller sensitivity for protons.

The CR39 plates were etched in a $6.25 \mathrm{~N} \mathrm{NaOH}$ solution. The $500 \mathrm{~mL}$ etchant material was contained in a cylinder $9 \mathrm{~cm}$ in diameter and $12 \mathrm{~cm}$ high (the etchant material extended only to $7.9 \mathrm{~cm}$ height), and was heated using a hot plate. The temperature was monitored on-line, right next to the small (less than $6 \mathrm{~mm}$ diameter) irradiated area of the CR39. During

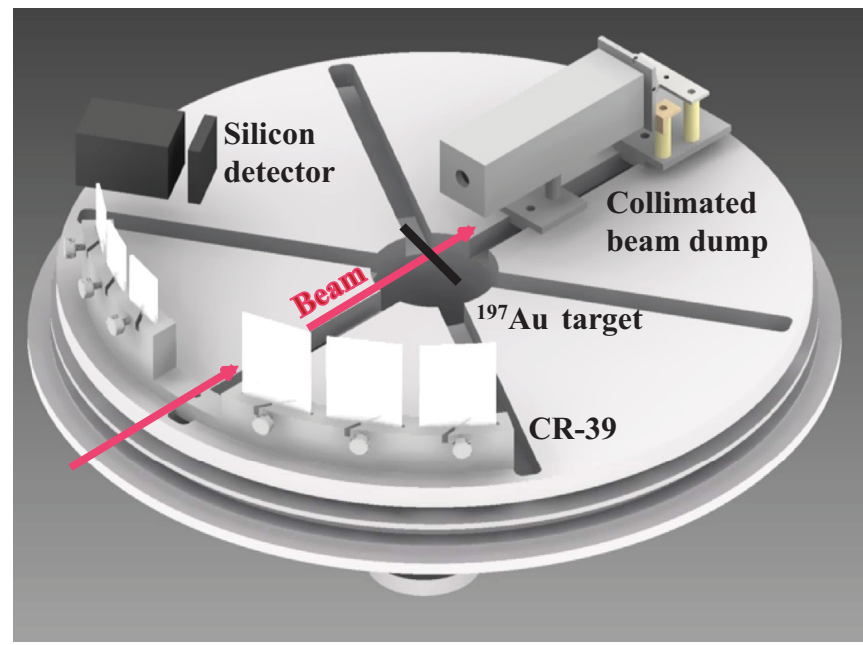

FIG. 1. Schematic diagram of the experimental setup used at the TUNL at Duke University and at the $3 \mathrm{MV}$ van de Graaff accelerator at the WIS. This CAD drawing is showing to scale all components inside the scattering chamber (with a diameter of $30 \mathrm{~cm}$ ), depicting to scale the dimensions of the CR39 plates: Homalite plates are on beam right at $165^{\circ}, 149^{\circ}$, and $133^{\circ}$ wrt to the beam and TASL plates are on beam left at $167^{\circ}, 156^{\circ}$, and $145^{\circ}$ wrt to the beam.

all etching operations we did not observe temperature variations larger than $1{ }^{\circ} \mathrm{C}$ across the 6-mm diameter active area of the CR39. As we discuss later, temperature variations define the systematic error of our measured pit radii. The etchant volume was not stirred since we observed large temperature variation which are most likely due to nonlaminar turbulent flow caused by the stirring.

CR39 NTD are commonly etched in temperatures ranging between 60 and $90^{\circ} \mathrm{C}[17,18]$. TASL [16] discusses an analysis of pits from etching at $98^{\circ} \mathrm{C}$ (and they also recommended etching at $98^{\circ} \mathrm{C}$ ). The MIT group published results from etching at $85^{\circ} \mathrm{C}$ [19]. We chose to etch for 30 minutes at $90^{\circ} \mathrm{C}$. This rather high etching temperature requires extra care as we discuss below. Under these etching conditions, micron-sized circular pits were produced after 30 minutes that were imaged with a microscope.

The bulk etch rate was measured by weighing a nonirradiated CR39 before and immediately after etching and washing in desalinated water. We measured at this rather high temperature the large bulk etch rate of $\sim 13 \pm 2 \mathrm{micron} / \mathrm{h}$, in agreement with published measurements $[16,18,20]$. We note that the absorption of water during the etching process may indeed contribute to a systematic bias in our measurement of the bulk etch rate. But the agreement with other measurements may indicate a common systematic error, or perhaps that water absorption is small in our etch condition (e.g., high temperature).

The measured bulk etch rate is not expected to be altered by the ambient gamma-ray (and X-ray) background at the SARAF, which is well below 0.1 MRad. However, it is quite possible that the large gamma flux contributes to the background observed at radii considerably smaller than 1 micron. Such a high density of pits with very small radii was observed when CR39 were exposed to low-energy electrons [21]. The 
temperature of the experimental setup while the LiLiT target is on is well below $60^{\circ} \mathrm{C}$, and it does not affect the bulk etch rate. All measurements performed in a vacuum in this study were very short, at most 15 to 30 minutes. Hence, issues due to outgassing are expected to be small; for example, the MIT group [22] reported no effect due to pre-outgassing before the irradiation over 16 hours, and post-outgassing up to 68 hours. All of our measured data are for pits densities which are well below the density that leads to overlapping pits; e.g., our Monte Carlo simulation for pits of radius of 1 micron with a pit density of 1920 per $\mathrm{mm}^{2}$ leads to a $1 \%$ overlap probability. However, all our data are measured with less than 500 pits per $\mathrm{mm}^{2}$ for a pit radius of 1 micron.

\section{B. Microscopy}

The etched CR39 detector plates used for the energy calibration measurement, were imaged with a Nikon TE2000 E fully automated inverted microscope set for brightfield imaging, controlled by NIS Elements software (version 4.3), either through a $40 \times / \mathrm{NA}=0.6 \mathrm{LWD}$ objective with correction collar set to zero cover-glass thickness, or using a $20 \times / \mathrm{NA}-0.4$ LWD objective with its correction collar set to zero cover-glass thickness. The $40 \times$ objective was used together with a QImaging Retiga 2000R cooled 2MP CCD camera that acquired the images coupled to the microscope with a $1 \times$ adapter. The $20 \times$ objective was used together with a Nikon DS-Qi2 monochrome 16MP CMOS imager coupled to the microscope through a $2.5 \times$ adapter. Large areas (typically $2 \times 2 \mathrm{~mm}$ ) that exceed the field of view (FOV) of the objective were captured by acquiring multiple fields with $10 \%$ overlap. To ensure that the best focus plane of the detector was captured over widely separated fields image stacks, consisting of 11 planes with an interplane spacing of $1.5 \mu \mathrm{m}$ (15 $\mu \mathrm{m}$ total depth), centered about the best focus plane of the pits, were acquired. A background illumination image with no detector inserted was also acquired for use in correction of illumination nonuniformity and fixed artifacts, as described below.

All subsequent image processing was done with the Fiji distribution [23] of ImageJ [24]. The preprocessing steps included the following.

(1) Correction of illumination nonuniformity and other fixed artifacts. This was done by dividing the detector images by the background image.

(2) A minimum intensity Z-projection of the stacks was done to take advantage of the fact that the pits appear as dark spheres when close to their best focus plane. The pits also have a second focus plane in which they focus the brightfield illumination to a bright spot. The minimum intensity projection enables us to ignore this phenomenon, but we note that it may prove useful in the future for characterization of the pits.

(3) Stitching of the multiple fields: Stitching was done with the Grid/Collection plugin [25] that is part of the FIJI distribution.

(4) The Threshold From Background function included with the BAR plugin collection [26] was used to segment the pits. The mean background gray level and background standard deviation were estimated based on a region of interest $(\mathrm{ROI})$ that covered $0.8 \times 0.8$ of the total image area

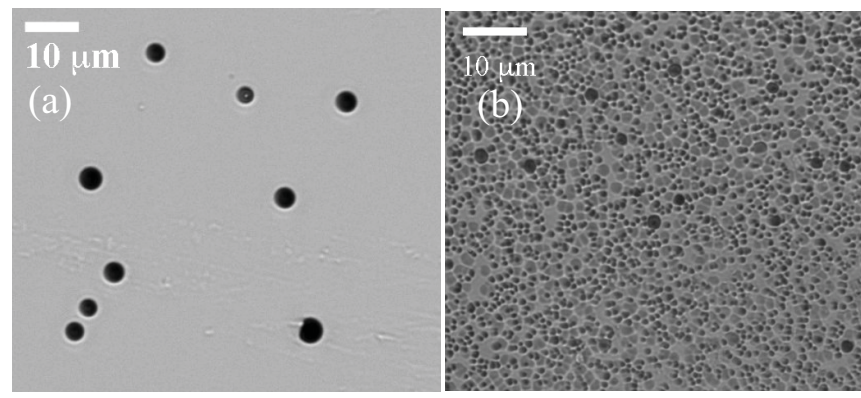

FIG. 2. (a) Typical pits observed after etching CR39 plates exposed to $3.18 \mathrm{MeV}$ alpha-particles from a ${ }^{148} \mathrm{Gd}$ radioactive source. (b) Typical pits observed in-beam using RBS of $1.5 \mathrm{MeV}$ protons. A scale of 10 microns is shown. The pits with very small radii are discussed in the text.

(i.e., $10 \%$ on each edge was not included). These criteria work because the objects are sparse so that the statistics of the image are essentially the background statistics. A small fraction of $10 \%$ on each edge was excluded because edges are where one may find edge artifacts, even though these edge artifacts are generally small. We also note that we have good statistics without the edges. The selection criteria for pits were as follows.

(a) Pixel intensity more than two standard deviations above the estimated background mean.

(b) Minimum area of 45 pixels (about $1.57 \mu \mathrm{m}^{2}$ ). For a round pit this would imply a minimum radius of $0.71 \mu \mathrm{m}$.

(c) Minimum circularity and roundness of 0.2 and 0.3 , respectively. An object's circularity in ImageJ is defined as $4 \pi\left(\right.$ area/perimeter $\left.{ }^{2}\right)$ [27]. An object's roundness in ImageJ is a measure of the ratio of the minor to major axes of the best-fit ellipse (based on matching the first two moments of the shape [28]) for that object. Both of these functions serve to filter out artifacts that are highly noncircular.

The extended particle analyzer included with the BIOVOXXEL TOOLBOX [29] filtered on size, circularity, and roundness, and provided measurements of any required morphological parameters such as area or perimeter.

Typical microscope pictures $(\times 40$ magnification) of pits obtained during the alpha-particle energy calibration measurements and for the $1.5 \mathrm{MeV}$ proton energy calibration are shown in Fig. 2. Note that we only scan an area with a diameter of a few $\mathrm{mm}$ in the CR39. The plates were placed at distances of at least $5 \mathrm{~cm}$ from the source, hence our energy calibration measurements are for charged particles that enter the NTD perpendicular to the surface, leading to the observed circular pits.

\section{Measured energy calibration spectra}

The spectra of observed radii from alpha-pits with energy of $3.18 \mathrm{MeV}$ (from the ${ }^{148} \mathrm{Gd}$ source) and $1.4 \mathrm{MeV}$ (from RBS) are shown in Fig. 3. We also show the spectrum of radii observed for $1.5 \mathrm{MeV}$ protons (from RBS) extending from the threshold at 0.8 microns to 1.4 microns. NTD are well known to have intrinsic background inherent to the CR39 plate itself $[11,30]$. This inherent noise is similar to the electronic noise 


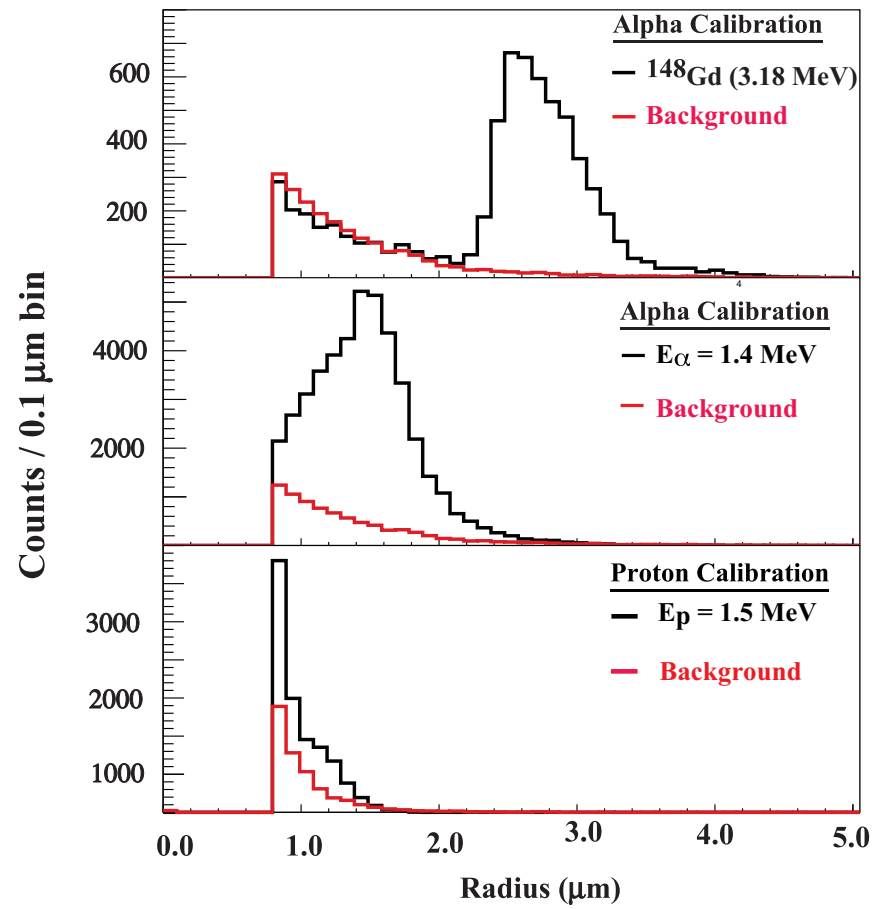

FIG. 3. The energy calibration of CR39 plates: We show the measured radii of pits in $6.983 \mathrm{~mm}^{2}$ from irradiation with alphaparticles from a ${ }^{148} \mathrm{Gd}$ source $(3.18 \mathrm{MeV})$ and RBS (1.4 MeV), and the measured radii of pits observed in $4.219 \mathrm{~mm}^{2}$ from irradiation with protons $(1.5 \mathrm{MeV})$. The exponential backgrounds below $1.5 \mathrm{mi}-$ crons (shown in red) were measured behind a 50- $\mu \mathrm{m}$-thick aluminum foil that stopped the alpha-particles and protons.

observed with silicon detectors, and in both cases this noise is cut out of the observed spectra.

Note that the measured line shape of the CR39 detector response for mono-energetic alpha-particle is asymmetric with tails both on the low and high radii sides. This asymmetric shape is most likely due to the fact that pits of particle with lower energy, develop in contrast before they start developing in diameter, leading to an asymmetric distribution when plotted on a diameter scale (see the Appendix and Fig. 1(c) of [19]).

The absolute detection efficiency of the CR39 plates for detecting $3.18 \mathrm{MeV}$ alpha-particles was measured using a $100 \mathrm{nCi}$ calibrated spectroscopic ${ }^{148} \mathrm{Gd}$ source (absolute activity $\pm 3.2 \%)$ that was placed at a distance of $153( \pm 2) \mathrm{mm}$. Pits in an area of a few $\mathrm{mm}^{2}$ in the CR39 plate were measured to yield the absolute efficiency of $93.3 \% \pm 3.8 \%$ (stat) $\pm 3.2 \%$ (calib) $\pm 3.5 \%$ (background). With a total uncertainty of $6.1 \%$, we conclude that the absolute detection efficiency of recording pits from $3.18 \mathrm{MeV}$ alpha-particle is close to $100 \%$. In the Weizmann measurement setup, shown in Fig. 1, we used a silicon detector to measure the RBS from the gold foil and measure the proton pit efficiency of $8.7 \pm 1.3 \%$. The small efficiency observed by us is most likely due to the short etching time at high temperature, and is similar to the observation of the MIT group [31] who emphasized that a short time interval while allowing for "less crowded" plates may yield small proton efficiency. However, no quantitative comparison between the two measurements can be carried out since the MIT group used TASL plates and we use Homalite plates. Indeed, the use of CR39 plates from the same source and the same batch has been emphasized before [32].

We compare our measured pit diameter shown in Fig. 3 to a previous measurement [15] using the scaling law discussed in [17]. But one must be careful to compare pits with radii on the same side of the Bragg-like curve. Hence, we compare our result to the radii obtained during 14 hours of etching shown in Fig. 2 of [15]. Our etch length over half an hour at $90{ }^{\circ} \mathrm{C}$ is: $L_{\text {Etch }}=(13 \pm 2) \times 0.5=6.5 \pm 1.0$ microns. For the etching performed over 14 hours at $70^{\circ}$ [15] we calculate [20] $L_{\text {Etch }}=(2.3 \pm 0.5) \times 14=32.2 \pm 7.0$ microns. Hence using the measured pit diameter (at $70^{\circ}$ ) of $3.2 \mathrm{MeV}$ alpha-particles of $\sim 30$ microns shown in Fig. 2 of [15] we predict for our etch conditions $\left(\right.$ at $90^{\circ}$ ) the radius of $\frac{6.5}{32.2} \times 15=3.03 \pm 0.7$, which is in good agreement with our measured pit radius of $\sim 2.55$ microns for the ${ }^{148} \mathrm{Gd}$ data, shown in Fig. 3 .

Furthermore, for an exposure of $3.18 \mathrm{MeV}$ alpha-particle from ${ }^{148} \mathrm{Gd}$ during the research and development phase of this study, we chose to etch the CR39 plate at $70^{\circ}$ over one and a half hour (the so produced spectrum of radii was shown at the Nuclei in the Cosmos 2016 conference [4,33]). Under this etching condition we observed the maximum yield at a radius of $1.7 \pm 0.2$ micron. At $70^{\circ}$ the measured bulk etch [20] leads to $L_{\text {Etch }}=(2.3 \pm 0.5) \times 1.5=3.45 \pm 0.75$ microns. The observed smaller radius of 1.7 micron at $70^{\circ}$ is $66 \%$ of the 2.55 micron radius observed with the $90^{\circ}$ etching, as shown in Fig. 3. It is in agreement with the predicted " $L_{\text {Etch }}$ scaling": $\frac{(3.45 \pm 0.75)}{(6.5 \pm 1.0)}=0.53 \pm 0.14$.

The high etching temperature leads to the large bulk etch rate that is agreement with previous data [16,18,20]. However, it is useful to estimate the conditions under which for example, a pit with a diameter of 1.5 micron will be extended by additional 0.2 micron; the systematics uncertainty of our measurement. We use the scaling parameter $L_{\text {etch }}=V_{b} \times \Delta t$ introduced in [17] to estimate this systematic uncertainty. The bulk etch velocity $\left(V_{b}\right)$ is [34]

$$
V_{\text {bulk }}=\mathrm{f}_{B} C^{n_{B}} e^{-\epsilon / k T},
$$

where $T$ is the temperature in $\mathrm{K}, C$ is the concentration of the etchant in the unit of normality, and $k$ is the Boltzman's constant. The constants $f_{B}, n_{B}$, and the activation energy $\epsilon$ were obtained from fitting procedures [34] as $\mathrm{f}_{B}=(8.2 \pm$ $0.4) \times 10^{11} \mu \mathrm{m} / \mathrm{h}, \mathrm{n}_{\mathrm{B}}=3 / 2$ and $\epsilon=0.88 \pm 0.04 \mathrm{eV}$. Note that using Eq. (1) we predict for our etching conditions the central value of the bulk etch rate of $9.16 \mu \mathrm{m} / \mathrm{h}$, very close to our measured value of $13 \pm 2 \mu \mathrm{m} / \mathrm{h}$ (when including the stated error-bar in $\epsilon$ [34], the range of predicted bulk etch rate is in good agreement with our measured value).

We deduce from Eq. (1) that a 0.2 micron shift for a pit radius of 1.5 micron will require 4 minutes additional etch time beyond the 30 minutes used by us, an increase in the normality by 0.54 beyond the 6.25 used by us, and an increase in the temperature by $1.7^{\circ} \mathrm{C}$ beyond the $90^{\circ}$ used by us. Such variations across a few $\mathrm{mm}$ in one CR39 plate are not possible within our strict etching procedures. However, a variation between two differently etched CR39 plates by $1.7^{\circ} \mathrm{C}$ cannot be ruled out. Hence, we quote the systematic uncertainty of $0.2 \mu \mathrm{m}$ in deducing our measured pit radii. 


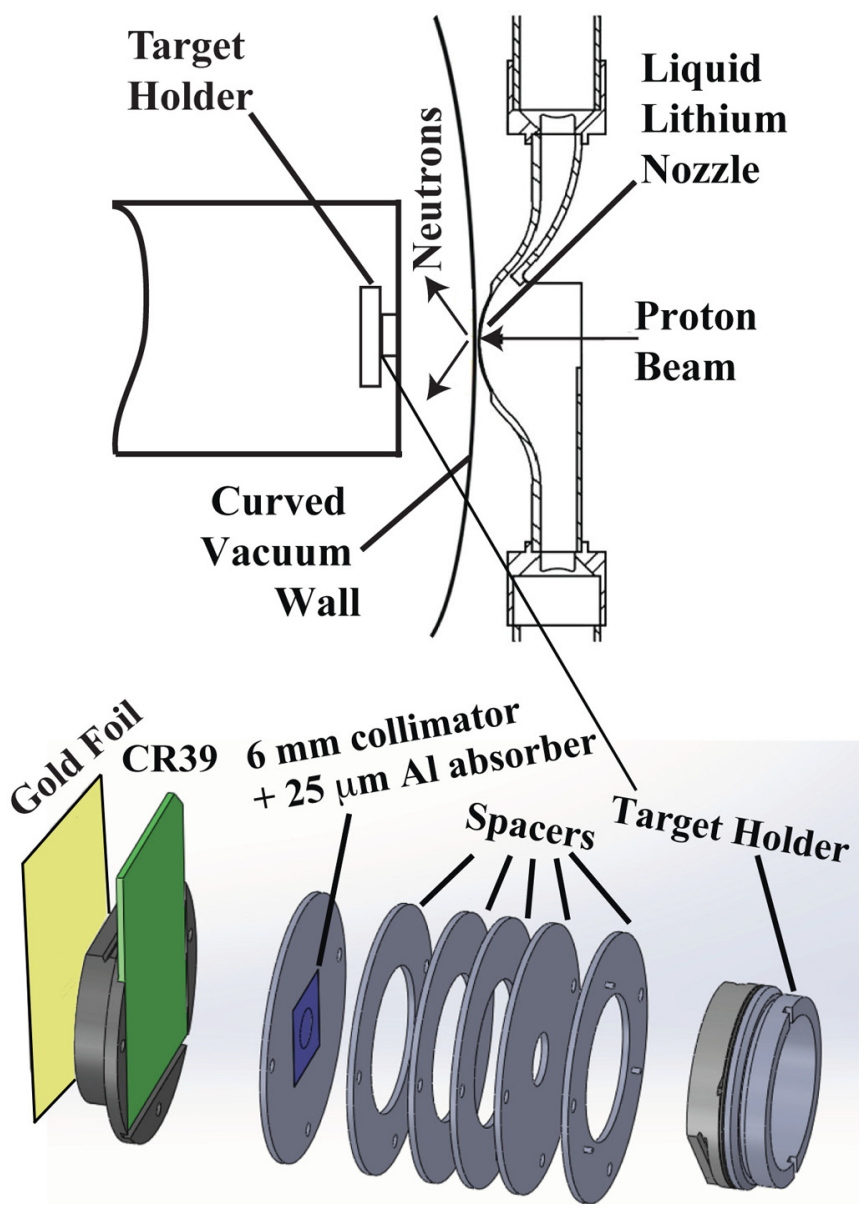

FIG. 4. Schematic diagram of the experimental setup used at the SARAF including the secondary $\left({ }^{7} \mathrm{Be}\right)$ target holder and the primary LiLiT target [6]. The gold foil used to measure the total beam fluence is shown (schematically) behind the CR39 plate. The detected charged particles are confined to $\pm 30^{\circ}$ wrt to the neutron beam.

\section{Epithermal neutrons: (In-Beam) Background measurement}

An in-beam background measurement with a CR39 plate was performed at SARAF with the experimental setup shown in Fig. 4. This setup was also used for measurements of protons and alpha-particles from the interaction of neutrons with ${ }^{7} \mathrm{Be}$ targets, and it is essential to measure the in-beam induced background of this setup. As we demonstrate below, in this measurement we only measure the interaction of epithermal neutrons with the CR39 plate.

The neutron beams were produced by bombarding LiLiT [5,7] with a 1 to $2 \mathrm{~mA}$ proton beam for a total of $3.11 \mathrm{~mA}$-hr with a proton energy $E_{p}=1.935 \mathrm{MeV}$ and energy spread of $\pm 15 \mathrm{keV}$. The resulting neutrons are confined to the forward angles $\left(\theta \leqslant 60^{\circ}\right)$ with a quasi-Maxwellian energy distribution [8,9]. The small "neutron angles" utilized in this measurement yield the rather high "effective temperature" $k T=49.5 \mathrm{keV}$, shown in Fig. 1 of [2]. The integrated beam luminosity was measured with a fission chamber (not shown in Fig. 4) and by placing a gold foil directly behind the detector setup, as shown in Fig. 4, and then measuring the

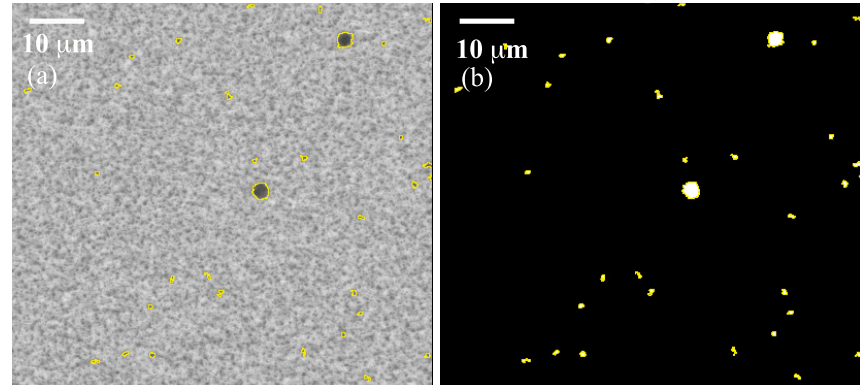

FIG. 5. (a) Typical pits observed with epithermal neutronbeamss leading to only circular pits. The scale of 10 microns is shown. The region of interest (ROI) detected by the segmentation algorithm is shown with yellow circumference. (b) Binary plot of pits selected by the segmentation algorithm. Note the irregular shape small radii pits $(\leqslant 0.8$ micron) produced by the overlap of smaller radii pits. Smaller objects cannot be considered significant due to the high level of background in that size range.

accumulated activity of the $412 \mathrm{keV}$ line from the ${ }^{197} \mathrm{Au}(n, \gamma)$ reaction with a well-known energy-dependent cross section.

The CR39 NTD were placed behind a 6-mm diameter collimator made of 0.5 -mm-thick pure $5 \mathrm{~N}$ aluminum that was placed at a distance of $7 \mathrm{~mm}$ from the target holder (see Fig. 4). Areas smaller than $11.4 \mathrm{~mm}^{2}$ (3.81-mm diameter) out of the available $28.3 \mathrm{~mm}^{2}(6.0-\mathrm{mm}$ diameter) were used to analyze pits in the NTD to stay clear of the edge of the collimator (fiducial area).

The CR39 plates used in the in-beam measurement were etched and analyzed using the exact same procedure as for the calibration measurement. A $z=-4$ image slice is shown in Fig. 5. We only observe circular pits for the data measured with epithermal neutrons, indicating tracks that are in the direction of the neutron beam, perpendicular to the surface of the NTD, as was the case for the calibration data. We also note the high density of pits with small radii below 0.8 microns, observed in the neutron in-beam measurement, which resembles the high density of pits with small radii observed in the proton calibration shown in Fig. 2.

\section{E. Epithermal neutrons results}

The spectrum of radii measured in the neutron in-beam experiment are shown in Fig. 6, where we observe the same exponential rise toward low radii below 1.4 micron, as observed in the background of the calibration data shown in Fig. 3, albeit with different slopes (on semilog plots). This background is most likely intrinsic to the CR39 plate [11,30]. The spectrum of radii above 1.4 microns, is distinctly different below 1.4 micron, as noted by the straight line added to the data below 1.4 micron in Fig. 6 .

In our attempt to understand the region of radii of interest (RRI) 1.5 to 3.4 microns shown in Fig. 6 we consider all possible charged particles produced by neutron interactions with all materials included in the experimental setup shown in Fig. 4. We conclude that only the ${ }^{17} \mathrm{O}(n, \alpha)$ reaction with $Q$-value $=+1.82 \mathrm{MeV}$, and the ${ }^{14} \mathrm{~N}(n, p)$ reaction with $Q$ value $=+0.625 \mathrm{MeV}$, are energetically possible. The small amount of ${ }^{14} \mathrm{~N}$ in our setup, as well as our etching condition 


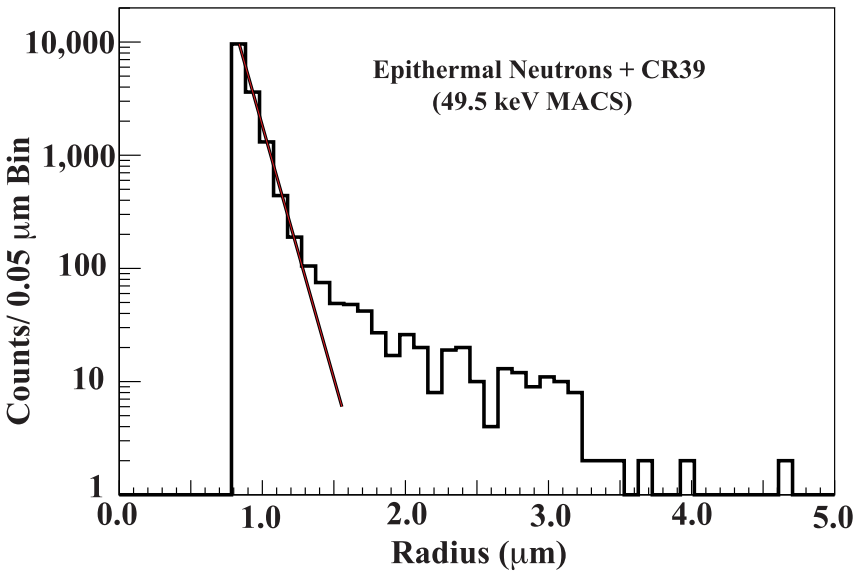

FIG. 6. The radii of pits measured over $11.373 \mathrm{~mm}^{2}$ from the in-beam SARAF measurement with epithermal neutrons. A line indicating the slope below 1.4 micron is added to guide the eye.

that inhibit the formation of proton pits, lead to a negligible background of proton-pits above 1.4 microns. However, the pits from the combined alpha-particles and ${ }^{14} \mathrm{C}$, which are emitted back to back in the ${ }^{17} \mathrm{O}(n, \alpha){ }^{14} \mathrm{C}$ reaction $\left(1<E_{n}<\right.$ $180 \mathrm{kev}$ ), with total energy of $\sim 2 \mathrm{MeV}$, can clearly contribute above 1.4 microns.

\section{F. Cold neutrons: Background measurement}

In an attempt to understand the "excess counts" in the RRI above 1.4 micron, a bare CR39 NTD was irradiated with cold neutrons at the Institut Laue Langevin (ILL) at Grenoble, France, with a total thermal equivalent fluence of $2.0 \times 10^{13} \mathrm{n} / \mathrm{cm}^{2}$. These cold neutrons $(\sim 4 \mathrm{meV})$ cannot break molecular bonds (obviously, CR39 is stable at room temperature), and hence the CR39 NTDs are not sensitive to the direct cold neutron beam. Therefore, no significant ionizing radiation exists in the ILL cold neutron beam and the tracks in the CR39 must be produced inside the CR39 $\left(\mathrm{C}_{12} \mathrm{H}_{18} \mathrm{O}_{7}\right)$ by the interaction of the cold neutrons with carbon, oxygen, and hydrogen atoms of the CR39 plate. In particular the ${ }^{17} \mathrm{O}(n, \alpha)$ reaction and the ${ }^{1} \mathrm{H}(n, \gamma)$ reaction with thermal cross sections of 257 and $348 \mathrm{mb}$, respectively, are of interest. Since gamma rays do not interact efficiently with CR39, in the ILL data we only observe pits produced by $\alpha+{ }^{14} \mathrm{C}$ from the ${ }^{17} \mathrm{O}(n, \alpha){ }^{14} \mathrm{C}$ reaction that occur inside the CR39 plate.

The ${ }^{17} \mathrm{O}(n, \alpha)$ reaction inside $\mathrm{CR} 39$ plates was mentioned before [35] as possible background, but so far no direct evidence was found. The importance of the ${ }^{17} \mathrm{O}(n, \alpha)$ reaction as a candidate for the source of background necessitated the irradiation of a bare CR39 plate with cold neutron at the ILL. The ILL CR39 plates were etched using the exact same procedure as for the plates from the SARAF measurement and a picture of the obtained pits is shown in Fig. 7. Note the large number of ellipsoidal pits observed in the ILL irradiation unlike the case of the SARAF measurement where we observed only circular pits as shown in Fig. 5. As we demonstrate below the ellipsoidal pits are a consequence of the geometry of the tracks due to the kinematics of the cold neutrons in the ${ }^{17} \mathrm{O}(n, \alpha)$ reaction.

\section{G. Cold neutron results}

The pits from the ILL irradiation were analyzed using the same algorithm as for the SARAF measurement, with the condition requiring circularity being relaxed. The obtained radii are shown in Fig. 7. The $Q$-value of the ${ }^{17} \mathrm{O}(n, \alpha)$ reaction is $1.82 \mathrm{MeV}$ leading to the energy deposited by the combined alpha-particle $(1.41 \mathrm{MeV})$ and ${ }^{14} \mathrm{C}(0.41 \mathrm{MeV})$, which are emitted back to back. The $1.41 \mathrm{MeV}$ alpha-particles and $0.41 \mathrm{MeV}{ }^{14} \mathrm{C}$ have a range of 5.9 and 1.2 micron in CR39, respectively, and the corresponding $\mathrm{dE} / \mathrm{dx}$ of the ${ }^{14} \mathrm{C}$ which is 1.7 times that of the alpha-particle; i.e., 411 versus $239 \mathrm{keV} / \mu \mathrm{m}$. Hence the radiation damage produced by the ${ }^{14} \mathrm{C}$ is considerably larger and more localized. Unfortunately, no calibration of pits produced by ${ }^{14} \mathrm{C}$ could be obtained in this study, but it appears reasonable to consider the "equivalent alpha-particle energy" that would produce the same radiation damage as ${ }^{14} \mathrm{C}$ to be $1.7 \times 0.4=0.68 \mathrm{MeV}$, leading to alpha-particle total "equivalent energy" deposit of approximately $2.1 \mathrm{MeV}$.

For slow (cold) neutron capture, the kinetic energies of the ejected particles are dominated by the $Q$ value of the reaction.
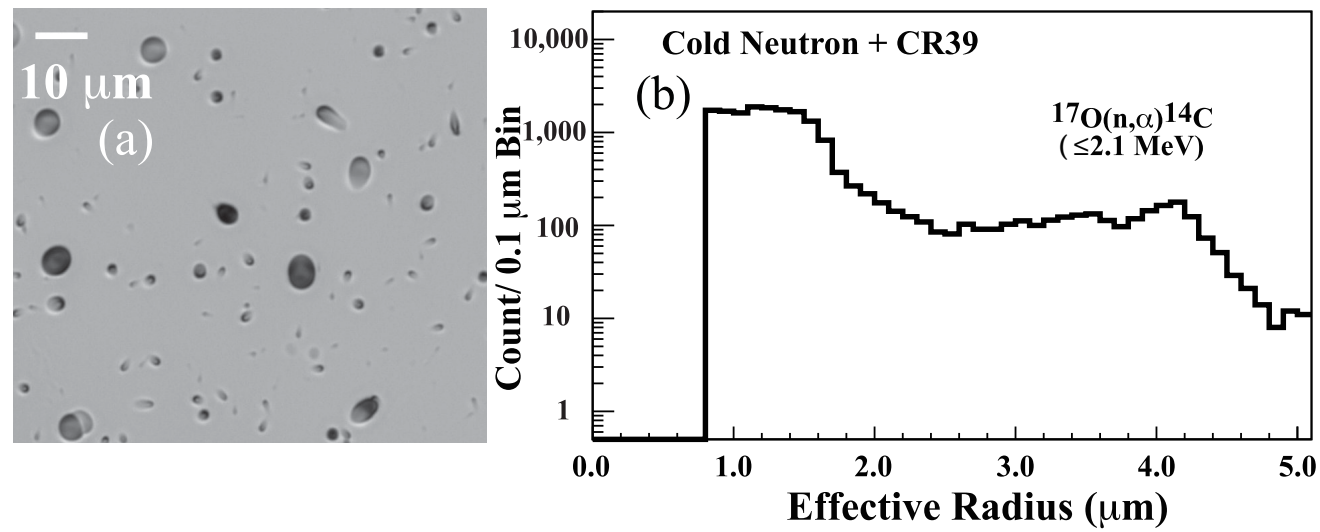

FIG. 7. (a) Typical pits observed with cold neutrons impinging on a bare CR39 plate. A scale of 10 microns is shown. Note the large number of ellipsoidal pits. (b) The measured "effective radii" of pits observed in $4.435 \mathrm{~mm}^{2}$ from $1.82 \mathrm{MeV}$ deposited by $\alpha+{ }^{14} \mathrm{C}$ from the ${ }^{17} \mathrm{O}(\mathrm{n}, \alpha)$ reaction inside the $\mathrm{CR} 39$ plate; note the large number of pits with large "effective radii" above 3.4 microns. 
At these energies the ${ }^{17} \mathrm{O}(n, \alpha)$ reaction is also an $s$-wave interaction. The kinematics of the reaction with cold neutron yield charged particles which are emitted isotropically from the compound nucleus. The large number of ellipsoidal pits observed in the ILL data, shown in Fig. 7, are produced by tracks oriented at a finite angle with respect to the surface of the NTD. We note that tracks parallel to the surface produce very shallow pits that are etched out due to bulk etching. The area $(A)$ of the pit image extracted by ImageJ was used to extract radii for the circular pits. For the ellipsoidal pits we extract "effective radii" $(R)$, where $A=\pi R^{2}$, and the extracted radii $(R)$ are shown in Fig. 7.

In contrast the interaction of the epithermal neutrons with ${ }^{17} \mathrm{O}$ is governed by the $p$-wave $J^{\pi}=1^{-}$resonance state [36] and the energy (and momentum) of the incident neutrons (up to $180 \mathrm{keV}$ ) is no longer negligible. This leads to a kinematical focusing and forward peaked angular distribution with tracks perpendicular to the surface of the CR39 plate. Hence, we observe a lack of ellipsoidal pits in the SARAF measurement, as shown in Fig. 5. At large radii, the difference between the mostly ellipsoidal pits observed in the ILL irradiation and the circular pits observed at the SARAF measurement, is due to the difference in the ${ }^{17} \mathrm{O}(n, \alpha)$ reaction kinematics that leads to isotropic distribution (of the emitted alpha-particles) for cold neutrons and forward peaked distribution (of the emitted alpha-particles) for the epithermal neutrons.

We emphasize that our alpha-particle energy-calibration shown in Fig. 3 must be used with caution due to the occurrence of tilted tracks that lead to ellipsoidal pits with larger projected "effective radius." Our calibration was measured for alpha-particles that penetrate perpendicular to the surface and form only circular pits with smaller radii. In addition a slight difference between the vacuum conditions at ILL and vacuum conditions of our calibration setup may lead to slightly different calibration due to outgassing [22].

The two spectra measured at SARAF (Fig. 6) and ILL (Fig. 7) show distinct shoulder-like edges at 3.4 and $4.8 \mathrm{mi}-$ crons, respectively. It appears that the RRI above 1.4 micron in the SARAF spectrum has been stretched in the ILL spectrum by a factor of $\sqrt{2}$. Indeed, when requiring pit circularity (thus removing the ellipsoidal pits), we observe the shoulder at the same radius of 3.4 microns, as we discuss below. Hence, we chose to compress the ILL spectrum by dividing the ILL radii by $\sqrt{2}$, and we discuss the obtained spectrum below.

\section{COMPARISON OF THE ILL AND SARAF MEASUREMENTS}

The excess pits observed in the ILL cold neutron data, shown in Fig. 7, in the region of "effective radii" of 3.4 to 4.8 micron arise from ellipsoidal pits. These large "effective radii" are a result of the geometric projection that leads to ellipsoidal pits, which are not present in the SARAF data, shown in Fig. 6. A complete understanding of this geometrical effect requires extensive Monte Carlo simulations that are beyond the scope of this report. Still, simple geometrical considerations of the tilt angle of the ellipsoidal pits leads to a stretching factor close to $\sqrt{2}$. In the discussion below, we develop a few of the concepts that will be included in such Monte Carlo simulations.

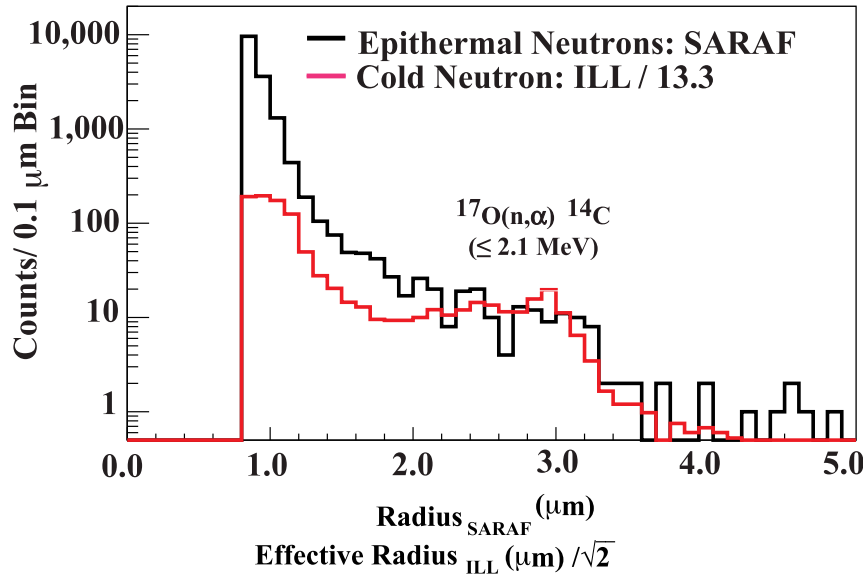

FIG. 8. A comparison of the yield measured at SARAF (black) shown in Fig. 6 and at ILL (red) shown in Fig. 7. The ILL effective radii are divided by $\sqrt{2}$, as discussed in the text. The ILL yields are divided by 13.3 to account for different neutron fluencies and the cross sections of the ${ }^{17} \mathrm{O}(\mathrm{n}, \alpha)$ reaction. The normalized ILL spectrum (radius and yield) is shown in units of the SARAF spectrum.

The thermal neutron cross section of the ${ }^{17} \mathrm{O}(n, \alpha)$ reaction is known to be $257 \mathrm{mb}$, and for $50 \mathrm{keV}$ epithermal neutrons it is measured to be $3.0 \pm 0.15 \mathrm{mb}$ [37]. The thermal equivalent neutron fluence used in the ILL measurement is $2 \times 10^{13} \mathrm{n} / \mathrm{cm}^{2}$ and the neutron fluence measured by the gold foil in the SARAF measurement is $4.99 \times 10^{13} \mathrm{n} / \mathrm{cm}^{2}$. The CR39 area examined in the ILL measurement is $4.434 \mathrm{~mm}^{2}$ and in the SARAF measurement the scanned area is 11.373 $\mathrm{mm}^{2}$. Thus the total integrated cold neutron-count is $8.87 \times$ $10^{11}$ for the ILL measurement and $5.67 \times 10^{12}$ for the SARAF measurement. The number of interactions is proportional to the total neutron-number times the cross section, which leads to the ratio of interactions of ILL/SARAF $=13.3$.

Since the etching conditions of the ILL and SARAF measurements are identical, we probe the same depth of the CR39 and include the same areal density of ${ }^{17} \mathrm{O}$ "target nuclei." Therefore, we divide by 13.3 the observed yield of the ILL spectrum that includes the region of interest with alpha-particle signals from the ${ }^{17} \mathrm{O}(n, \alpha)$ reaction.

In Fig. 8 we show the ILL spectrum with the radius divided by $\sqrt{2}$ and the yield divided by 13.3. In the scaled RRI of 1.9 to 3.4 microns, where the ellipsoidal pits dominate, the two spectra are in agreement within the error bars of the shown data points. In this region we observe $203 \pm 14$ and $170 \pm 13$ counts in the SARAF measurement and the ILL measurement, respectively. Furthermore, in this region a fit of the SARAF data, using line shape of the ILL data (with effective radii compressed by $\sqrt{2}$ ), leads to $\chi^{2}=1.5$, indicating a common parentage for the two distributions. The low yield at radii above 3.4 microns is consistent with intrinsic background of the CR39 plates that was noted before [11,30]. Below 1.9 micron we do not observe the same simple scaling as would be expected for this region which is dominated by intrinsic noise of the CR39 plate.

In addition, in Fig. 9 we show the the ILL data with the requirement of circularity of the pits, thus removing the ellipsoidal pits. The obtained ILL yield is also divided by 


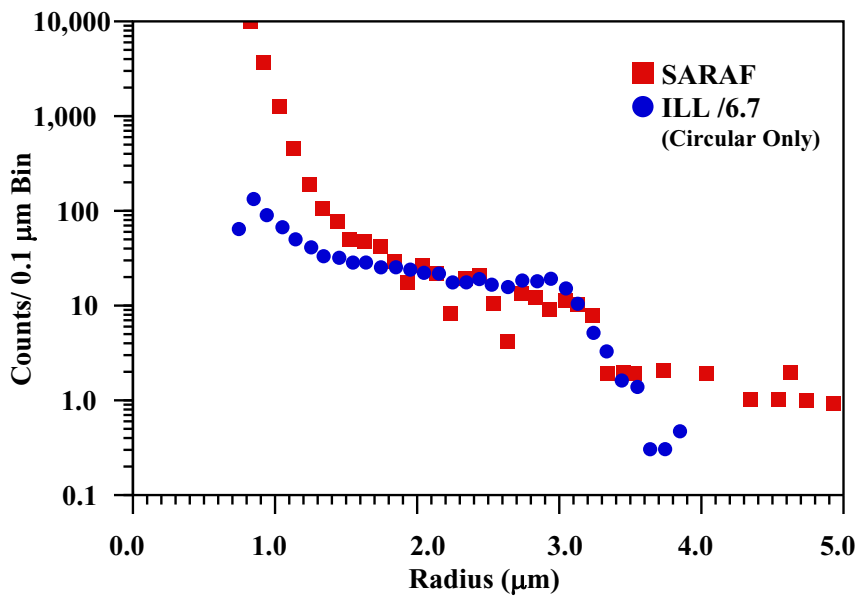

FIG. 9. A comparison of the yield measured at SARAF (red) shown in Fig. 6 and at the ILL (blue). For the ILL data we required circularity of the pits and the yield is divided by 6.7 .

6.7 and is compared to the SARAF spectrum shown Fig. 6. When removing the ellipsoidal pits from the ILL data with the 6.7 normalization factor, we obtain over the region of interest, a spectrum very similar to the one observed at SARAF, including the shoulder at 3.4 microns. In addition, the normalization factor of 6.7 , indicates that over the region of interest, approximately half of the pits in the ILL data are ellipsoidal.

The comparisons shown in Fig. 8 of the ILL data (Fig. 7) and the SARAF data (Fig. 6), and in Fig. 9, allow us to conclude that the region above 1.5 micron in the SARAF data is dominated by tracks from $\alpha+{ }^{14} \mathrm{C}$ emanating from the ${ }^{17} \mathrm{O}(n, \alpha)$ reaction with a total "equivalent energy" of up to $\sim 2.1 \mathrm{MeV}$. We provide here the first direct evidence for the ${ }^{17} \mathrm{O}(n, \alpha)$ interactions inside the CR39 plate. Since the interaction occurs inside the CR39 plates, the observed pits are for alpha-particles with energies of up to $\sim 2.1 \mathrm{MeV}$.
The observed background from the ${ }^{17} \mathrm{O}(n, \alpha)$ reaction, would be the limiting factor for measurements with CR39 plates of $(n, \alpha)$ reactions with low cross sections. But on the other hand, this "background line" of alpha-particles with energies up to $\sim 2.1 \mathrm{MeV}$, can be used for internal self calibration of measurements of large cross sections of $(n, \alpha)$ reactions, as we do in our measurement [2] of the cross section of the ${ }^{7} \mathrm{Be}\left(n, \gamma_{1}\right)^{*} \mathrm{Be}(3.03) \rightarrow \alpha+\alpha$, yielding alpha particles with energies up to $2.2 \mathrm{MeV}$.

\section{CONCLUSION}

In conclusion, we calibrated CR39 NTD with alphaparticles and protons of energies 1.4 to $3.18 \mathrm{MeV}$, and we studied with cold neutrons the background signals from the ${ }^{17} \mathrm{O}(n, \alpha)$ inside the CR39. We developed an etching procedure that is less efficient $(8.7 \%)$ for the formation of proton pits while fully developing $(\sim 100 \%)$ the alpha-pits. Using the ILL measurement with cold neutrons we demonstrate that the region of pits with radii above 1.5 micron is governed by the ${ }^{17} \mathrm{O}(n, \alpha)$ reaction inside the CR39 plates. Hence the ${ }^{17} \mathrm{O}(n, \alpha)$ reaction inside the CR39 would be the limiting factor in determining the sensitivity for measuring $\sim 2.1 \mathrm{MeV}$ alpha-particles from the $(n, \alpha)$ reactions. But, this "background line" of alpha-particles with energies of up to $\sim 2.1 \mathrm{MeV}$, can be used for internal self calibration of such measurements.

\section{ACKNOWLEDGMENTS}

We acknowledge discussions with Johan Frenje of MIT, Daniel Thomas Casey of the Lawrence Livermore National laboratory, and Daniel Berkovits of the SARAF. The material presented in this paper is based upon work supported by the U.S.-Israel Bi National Science Foundation, Award No. 2012098, and the U.S. Department of Energy, Office of Science, Nuclear Physics, Awards No. DE-FG02-94ER40870 and DE-FG02-91ER-40608.
[1] I. Mardor, O. Aviv, M. Avrigeanu, D. Berkovits, A. Dahan, T. Dickel, I. Eliyahu, M. Gai, I. Gavish-Segev, S. Halfon, M. Hass, T. Hirsh, B. Kaiser, D. Kijel, A. Kreisel, Y. Mishnayot, I. Mukul, B. Ohayon, M. Paul, A. Perry, H. Rahangdale, J. Rodnizki, G. Ron, R. Sasson-Zukran, A. Shor, I. Silverman, M. Tessler, S. Vaintraub, and L. Weissman, Eur. Phys. J. A 54, 91 (2018).

[2] M. Gai, E. E. Kading, M. Hass, K. M. Nollett, S. R. Stern, Th. Stora, and A. Weiss, Eur. Phys. J. Web Conf. 227, 01007 (2020).

[3] B. D. Fields, K. A. Olive, T.-H. Yeh, and C. Young, J. Cosmo. Astrop. Phys. 03 (2020) 010.

[4] E. E. Kading, Calibration of CR-39 Nuclear Track Detectors with Alpha-Particles and Protons for a Measurement of Neutron Interactions with 7Be and the Primordial 7Li Problem (2017), master's thesis, https://opencommons.uconn.edu/ gs_theses/1065/.

[5] G. Feinberg, M. Paul, A. Arenshtam, D. Berkovits, D. Kijel, A. Nagler, and I. Silverman, Nucl. Phys. A 827, 590c (2009).
[6] M. Paul, A. Arenshtam, S. Halfon, D. Kijel, M. Tessler, L. Weissman, D. Berkovits, Y. Eisen, I. Eliyahu, M. Friedman, G. Feinberg, A. Kreisel, I. Mardor, G. Shimel, A. Shor, and I. Silverman, J. Radioanal. Nucl. Chem. 305, 783 (2014).

[7] S. Halfon et al., Rev. Sci. Instr. 85, 056105 (2014).

[8] G. Feinberg, M. Friedman, A. Krasa, A. Shor, Y. Eisen, D. Berkovits, D. Cohen, G. Giorginis, T. Hirsh, M. Paul, A. J. M. Plompen, and E. Tsuk, Phys. Rev. C 85, 055810 (2012).

[9] M. Tessler et al., Phys. Lett. B 751, 418 (2015).

[10] J. A. Frenje, C. K. Li, F. H. Seguin, D. G. Hicks, S. Kurebayashi, R. D. Petrasso, S. Roberts, V. Yu. Glebov, D. D. Meyerhofer, T. C. Sangster, J. M. Soures, C. Stoeckl, C. Chiritescu, G. J. Schmid, and R. A. Lerche, Rev. Sci. Instr. 73, 2597 (2002)

[11] D. T. Casey, J. A. Frenje, F. H. Seguin, C. K. Li, M. J. Rosenberg, H. Rinderknecht, M. J.-E. Manuel, M. Gatu Johnson, J. C. Schaeffer, R. Frankel, N. Sinenian, R. A. Childs, R. D. Petrasso, V. Yu. Glebov, T. C. Sangster, M. Burke, and S. Roberts, Rev. Scient. Instr. 82, 073502 (2011). 
[12] Ch. Weiß et al. and The n_TOF Collaboration, Nucl. Instrum. Methods Phys. Res. A 732, 190 (2013).

[13] https://www.professionalplastics.com/professionalplastics/ content/downloads/HomaliteH-911_CR39.pdf.

[14] S. Halfon, M. Paul, A. Arenshtam, D. Berkovits, D. Cohen, I. Eliyahu, D. Kijel, I. Mardor, and I. Silverman, Appl. Rad. Isotopes. 88, 238 (2014).

[15] M. Sadowski, A. Szydlowski, M. Jaskola, and T. Czyzewski, Rad. Measur. 28, 207 (1997).

[16] http://www.tasl.co.uk.

[17] T. W. Jeong, P. K. Singh, C. Scullion, H. Ahmed, P. Hadjisolomou, C. Jeon, H. Yun, K. F. Kakolee, M. Borghesi, and S. Ter-Avetisyan, Sci. Rep. 7, 2152 (2017).

[18] E. M. Awad and H. M. El-Samman, Radiat. Meas. 31, 109 (1999).

[19] N. Sinenian, M. J. Rosenberg, M. Manuel, S. C. McDuffee, D. T. Casey, A. B. Zylstra, H. G. Rinderknecht, M. Gatu Johnson, F. H. Seguin, J. A. Frenje, C. K. Li, and R. D. Petrasso, Rev. Sci. Instrum. 82, 103303 (2011).

[20] E. M. Awad, V. Ditlove, M. Fromm, and D. Hermsdorf, Radiat. Meas. 44, 813 (2009).

[21] C. G. Wahl and J. G. McLean, Rad. Measur. 40, 43 (2005).

[22] M. J.-E. Manuel, M. J. Rosenberg, N. Sinenian, H. Rinderknecht, A. B. Zylstra, F. H. Seguin, J. Frenje, C. K. Li, and R. D. Petrasso, Rev. Sci. Instr. 82, 095110 (2011).

[23] J. Schindelin, I. Arganda-Carreras, E. Frise, V. Kaynig, M. Longair, T. Pietzsch, S. Preibisch, C. Rueden, S. Saalfeld, B. Schmid, J.-Y. Tinevez, D. J. White, V. Hartenstein, K. Eliceiri, P. Tomancak, and A. Cardona, Nature Methods 9, 676 (2012)

[24] C. A. Schneider, W. S. Rasband, and K. W. Eliceiri, Nat. Methods 9, 671 (2012).

[25] S. Preibisch, S. Saalfeld, and P. Tomancak, Bioinformatics 25, 1463 (2009).

[26] T. Ferreira, K. Miura, B. Chef, and J. Eglinger, Scripts: BAR 1.1.6, doi:10.5281/zenodo.28838, https://doi.org/10.5281/ zenodo.28838 (2015).
[27] W. Rasband, Circularity, https://imagej.nih.gov/ij/plugins/ circularity.html

[28] K. F. Mulchrone and K. R. Choudhury, J. Struct. Geol. 26, 143 (2004).

[29] J. Brocher, a BioVoxxel Toolbox, 2014, [Online], http://fiji.sc/ BioVoxxel_Toolbox.

[30] F. H. Seguin, J. A. Frenje, C. K. Li, D. G. Hicks, S. Kurebayashi, J. R. Rygg, B.-E. Schwartz, and R. D. Petrasso, S. Roberts, J. M. Soures, D. D. Meyerhofer, T. C. Sangster, J. P. Knauer, C. Sorce, V. Yu. Glebov, and C. Stoeckl, T. W. Phillips, and R. J. Leeper, Rev. Sci. Instr. 74, 975 (2003).

[31] M. J. Rosenberg, F. H. Seguin, C. J. Waugh, H. G. Rinderknecht, D. Orozco, J. A. Frenje, M. Gatu Johnson, H. Sio, A. B. Zylstra, N. Sinenian, C. K. Li, R. D. Petrasso, V. Yu. Glebov, C. Stoeckl, M. Hohenberger, T. C. Sangster, S. LePape, A. J. Mackinnon, R. M. Bionta, O. L. Landen, R. A. Zacharias, Y. Kim, H. W. Herrmann, and J. D. Kilkenny, Rev. Sci. Instr. 85, 043302 (2014).

[32] F. H. Seguin, N. Sinenian, M. Rosenberg, A. Zylstra, M. J.E. Manuel, H. Sio, C. Waugh, H. G. Rinderknecht, M. Gatu Johnson, J. Frenje, C. K. Li, R. Petrasso, T. C. Sangster, and S. Roberts, Rev. Sci. Instrum. 83, 10D908 (2012).

[33] E. E. Kading, M. Gai, M. Paul, T. Palchan, M. Tessler, R. Dressler, S. Heinitz, E. A. Maugeri, D. Schumann, M. Hass, I. Mukul, Y. Shachar, C. Seiffert, T. Stora, D. Ticehurst, and C. R. Howell, Proceedings of the 14th International Symposium on Nuclei in the Cosmos (NIC2016), Niigata, Japan, JPS Conf. Proc. Vol. 14 (J. Phys. Soc. Jpn., 2017).

[34] D. Nikezic and K. N. Yu, Mater. Sci. and Eng. R 46, 51 (2004).

[35] A. Seghour, F. Z. Seghour, and B. Saichi, Nucl. Instrum. Methods Phys. Res. A 589, 66 (2008).

[36] P. E. Koehler and S. M. Graff, Phys. Rev. C 44, 2788 (1991).

[37] H. Schatz, F. Kaeppeler, P. E. Kohehler, M. Wiescher, and H.-P. Trautvetter, Astr. Phys. Jour. 413, 750 (1993). 